\title{
Estimating the parameter of Exponential distribution under Type-II censoring from fuzzy data
}

\author{
Nayereh Bagheri Khoolenjani* \\ Department of Statistics, University of Isfahan, Isfahan,81746-73441, Iran \\ n.b.khoolenjani@gmail.com \\ Fateme Shahsanaie \\ Department of Statistics, Behbahan Khatam Alanbia University of Thechnology, Behbahan, Iran \\ shahsanaei@bkatu.ac.ir
}

Received 23 October 2015

Accepted 23 November 2015

\begin{abstract}
Statistical analysis of lifetime distributions under Type-II censoring scheme is based on precise lifetime data. However, in real situations all observations and measurements of Type-II censoring scheme are not precise numbers but more or less non-precise, also called fuzzy. This paper deals with the estimation of exponential mean parameter under Type-II censoring scheme when the lifetime observations are fuzzy and are assumed to be related to underlying crisp realization of a random sample. We use Newton-Raphson algorithm as well as EM algorithm to determine the maximum likelihood estimate (MLE) of parameter. We also obtain the estimate, via moment method and Bayesian procedure, of the unknown parameter. In addition, a new numerical method for parameter estimation is provided. Monte Carlo simulations are performed to investigate performance of the different methods. Finally, an example is presented in order to illustrate the methods of inference discussed here.
\end{abstract}

Keywords: Type-II censoring; Fuzzy lifetime data; Exponential distribution; Maximum likelihood estimation; Bayes estimator.

2000 Mathematics Subject Classification: 62N02, 62N86

\section{Introduction}

In life testing and reliability studies, the experimenter may not always obtain complete information on failure times for all experimental units. Data obtained from such experiments are called censored data. One of the most common censoring scheme is Type-II (failure) censoring, where the life testing experiment will be terminated upon the $r$-th ( $\mathrm{r}$ is pre-fixed) failure. This scheme is often adopted for toxicology experiments and life testing applications by engineers as it has been proven

${ }^{*}$ Corresponding author 
to save time and money. Several authors have addressed inferential issues based on Type-II censored samples; see for example, [13], [2], [19], [3], [9], [16], [11], [17], and [10]. Their research results for estimating parameters of different lifetime distributions under Type-II censoring are limited to precise data. However, in real situations all observations and measurements of continuous variables are not precise numbers but more or less non-precise. For instance, the lifetime observations may be reported as imprecise quantities such as: 'about $1000 h$ ', 'approximately $1400 h$ ', 'almost between $1000 h$ and $1200 h$ ', 'essentially less than $1200 h$ ', and so on. This imprecision is different from variability and errors. The best up-to-date mathematical model for this imprecision are so-called non-precise (fuzzy) numbers. Classical statistical procedures are not appropriate to deal with such imprecise cases. Therefore, we need suitable statistical methodology to handle these data as well.

In recent years, several researchers pay attention to applying the fuzzy sets to estimation theory. Huang et al. [8] proposed a new method to determine the membership function of the estimates of the parameters and the reliability function of multiparameter lifetime distributions. Coppi et al. [4] presented some applications of fuzzy techniques in statistical analysis. Pak et al. [14], [15] conducted a series of studies to develop the inferential procedures for the lifetime distributions on the basis of fuzzy numbers. In this paper, our objective is to devise the methods for parameter estimation regarding a life-test from which the Type-II censored data are reported in the form of fuzzy numbers. We analyze the data under the assumptions that the lifetimes of the test units are independent identically distributed exponential random variables. In Section 2, we first present in greater detail the problem addressed in this paper. Some preliminary concepts about fuzzy numbers is included in this section. In Section 3, we introduce a generalization of the likelihood function based on Type-II censoring from fuzzy data and then discuss Newton-Raphson algorithm as well as EM algorithm to determine the MLE of the unknown parameter. We then study the estimate via moment method and estimate via Bayesian procedure for the mean parameter. A new parameter estimation method, called 'computational approach estimation' (CAE), is also established. In Section 4, simulation study is carried out to assess the performance of the proposed methods and a real data from the life testing experiment is analyzed for illustrative purposes.

\section{Problem description}

Consider a reliability experiment in which $n$ identical units are placed on a life-test. Let $X_{1}, \ldots, X_{n}$ denote the lifetimes of these experimental units. We assume that these variables are independent and identically distributed as Exponential $E(\theta)$, with probability density function (pdf)

$$
f(x ; \theta)=\frac{1}{\theta} \exp \left(-\frac{x}{\theta}\right) \quad, x>0, \theta>0 .
$$

Let $X_{1: n} \leq X_{2: n} \leq \ldots \leq X_{n: n}$ denote the corresponding ordered lifetimes. Suppose the experimenter decides to carry out the life-test until the time of the $r$ th failure, then the data arising from such a life-test would be of the form $X_{1: n} \leq X_{2: n} \leq \ldots \leq X_{r: n}$ with the remaining $n-r$ lifetimes being more than $X_{r: n}$. This situation is referred to as Type-II censoring. We also denote the observed values of such a Type-II right censored sample by $x_{1: n}, \ldots, x_{r: n}$. Based on these observations, the likelihood function is given by:

$$
\begin{gathered}
L(\theta ; \mathbf{x})=\frac{n !}{(n-r) ! \theta^{r}} \exp \left(-\frac{\sum_{i=1}^{r} x_{i: n}+(n-r) x_{r: n}}{\theta}\right) . \\
\text { Published by Atlantis Press } \\
\text { Copyright: the authors } \\
182
\end{gathered}
$$


Thus the maximum likelihood estimator of $\theta$ can be obtained by

$$
\hat{\theta}=\frac{1}{r}\left(\sum_{i=1}^{r} x_{i: n}+(n-r) x_{r: n}\right)
$$

However, in many fields of application, it is sometimes impossible to obtain exact observations of lifetime. The obtained lifetime data may be imprecise most of the time. For instance, consider a life-testing experiment in which $n$ identical ball bearings are placed on test, and we are interested in the lifetime of these ball bearings. In practice, however, measuring the lifetime of a ball bearing may not yield an exact result. A ball bearing may work perfectly over a certain period but be braking for some time, and finally be unusable at a certain time. So, the number of revolutions to failure (in millions) for ball bearings may reported by means of the following imprecise quantities: "approximately lower than 45 ", "approximately 50 to 70 ", "approximately 75 ", "approximately 80 ", "approximately 90 to 100 ", "approximately higher than 120 ", and so on. In order to model imprecise lifetimes, a generalization of real numbers is necessary. These lifetimes can be represented by fuzzy numbers. A fuzzy number is a subset, denoted by $\tilde{x}$, of the set of real numbers (denoted by $\mathbb{R}$ ) and is characterized by the so called membership function $\mu_{\tilde{x}}($.$) . Fuzzy numbers$ satisfy the following constraints (see [5]):

(1) $\mu_{\tilde{x}}: \mathbb{R} \longrightarrow[0,1]$ is Borel-measurable;

(2) $\exists x_{0} \in \mathbb{R}: \mu_{\tilde{x}}\left(x_{0}\right)=1$;

(3) The so-called $\lambda$-cuts $(0<\lambda \leq 1)$, defined as $B_{\lambda}(\tilde{x})=\left\{x \in \mathbb{R}: \mu_{\tilde{x}}(x) \geq \lambda\right\}$, are all closed interval, i.e., $B_{\lambda}(\tilde{x})=\left[a_{\lambda}, b_{\lambda}\right], \forall \lambda \in(0,1]$.

Among the various types of fuzzy numbers, $L R$-type fuzzy numbers (the triangular and trapezoidal fuzzy numbers are special cases of the $L R$-type fuzzy numbers) are most convenient and useful in describing fuzzy lifetime data. Therefore, we shall focus on the set of $L R$-type fuzzy numbers.

Definition 2.1. (see [21] pp.62). Let $L$ (and $R$ ) be decreasing, shape functions from $\mathbb{R}^{+}$to $[0,1]$ with $L(0)=1 ; L(x)<1$ for all $x>0 ; L(x)>0$ for all $x<1 ; L(1)=0$ or $(L(x)>0$ for all $x$ and $L(+\infty)=0)$. Then a fuzzy number $\tilde{x}$ is called of $L R$-type if for $m, \alpha>0, \beta>0$ in $\mathbb{R}$,

$$
\mu_{\tilde{x}}(x)= \begin{cases}L\left(\frac{m-x}{\alpha}\right) & x \leq m \\ R\left(\frac{x-m}{\beta}\right) & x \geq m\end{cases}
$$

where $m$ is called the mean value of $\tilde{x}$ and $\alpha$ and $\beta$ are called the left and right spreads, respectively. Symbolically, the $L R$-type fuzzy number is denoted by $\tilde{x}=(\alpha, m, \beta)$.

Definition 2.2. Suppose that $\tilde{x}_{i}=\left(\alpha_{i}, m_{i}, \beta_{i}\right), i=1, \ldots, n$, be the $L R$-type fuzzy numbers. The fuzzy mean value of these numbers can be obtained as

$$
\overline{\widetilde{x}}=(\bar{\alpha}, \bar{m}, \bar{\beta}), \text { with } \bar{\alpha}=\frac{1}{n} \sum_{i=1}^{n} \alpha_{i}, \bar{m}=\frac{1}{n} \sum_{i=1}^{n} m_{i} \text { and } \bar{\beta}=\frac{1}{n} \sum_{i=1}^{n} \beta_{i}
$$

\section{Data, likelihood and parameter estimations}

In this section, we will first introduce a generalization of the likelihood function based on TypeII censoring when the lifetime observations are reported in the form of $L R$-type fuzzy numbers. 
The maximum likelihood estimate of the mean parameter will then be obtained using the NewtonRaphson as well as the EM algorithm in Section 3.2. The estimation, via moment method, Bayesian procedure and CAE method, of the unknown parameter will be discussed in Sections 3.3, 3.4 and 3.5 , respectively.

\subsection{Fuzzy lifetime data and the likelihood function}

Suppose that $n$ independent units are put on a test and that the lifetime distribution of each unit is given by (2.1). Now consider the problem where under the Type-II censoring scheme, failure times are not observed precisely and only partial information about them are available in the form of fuzzy numbers $\tilde{x}_{i}=\left(\alpha_{i}, m_{i}, \beta_{i}\right), i=1, \ldots, r$, with their corresponding membership functions $\mu_{\tilde{x}_{1}}\left(x_{1}\right), \ldots, \mu_{\tilde{x}_{r}}\left(x_{r}\right)$. Let the maximum value of the means of these fuzzy numbers to be $m_{(r)}$. The lifetime of $n-r$ surviving units, which are removed from the test after the $m$ th failure, can be encoded as fuzzy numbers $\tilde{z}_{r+1}, \ldots, \tilde{z}_{n}$ with the membership functions

$$
\mu_{\tilde{z}_{j}}(z)=\left\{\begin{array}{ll}
0 & z \leq m_{(r)} \\
1 & z>m_{(r)}
\end{array}, \quad j=r+1, \ldots, n .\right.
$$

The fuzzy data $\tilde{\mathbf{w}}=\left(\tilde{x}_{1}, \ldots, \tilde{x}_{r}, \tilde{z}_{r+1}, \ldots, \tilde{z}_{n}\right)$ is thus the vector of observed lifetimes. The corresponding observed-data log-likelihood function can be obtained, using Zadeh's definition of the probability of a fuzzy event (Zadeh [20]), as

$$
\begin{aligned}
L_{O}(\tilde{\mathbf{w}} ; \theta) & =\sum_{i=1}^{r} \log \int \frac{1}{\theta} \exp \left(-\frac{x}{\theta}\right) \mu_{\tilde{x}_{i}}(x) d x+(n-r) \log \left\{\int_{m_{(r)}}^{\infty} \frac{1}{\theta} \exp \left(-\frac{z}{\theta}\right) d z\right\} \\
& =-r \log \theta+\sum_{i=1}^{r} \int \exp \left(-\frac{x}{\theta}\right) \mu_{\tilde{x}_{i}}(x) d x-(n-r) \frac{m_{(r)}}{\theta} .
\end{aligned}
$$

\subsection{Maximum likelihood estimation}

The maximum likelihood estimate of the parameter $\theta$ can be obtained by maximizing the likelihood function (3.1). By taking the derivative of the log-likelihood (3.1) with respect to $\theta$, we have

$$
\frac{\partial}{\partial \theta} L_{O}(\tilde{\mathbf{w}} ; \theta)=-\frac{r}{\theta}+\sum_{i=1}^{r} \frac{\int \frac{x}{\theta^{2}} \exp \left(-\frac{x}{\theta}\right) \mu_{\tilde{x}_{i}}(x) d x}{\int \exp \left(-\frac{x}{\theta}\right) \mu_{\tilde{x}_{i}}(x) d x}+(n-r) \frac{m_{(r)}}{\theta^{2}}
$$

To achieve estimation via ML method, it is not easy to solve the equation $\frac{\partial}{\partial \theta} L_{O}(\tilde{\mathbf{w}} ; \theta)=0$, directly. In the following, Theorem 1 discusses the existence and uniqueness of the MLE of $\theta$.

Theorem 3.1. Under the Type-II censoring, the MLE of the mean parameter $\theta$ of an Exponential population exists and is unique.

Proof. Let $\lambda=\frac{1}{\theta}$. Due to the invariance property of MLEs, we will show the existence and uniqueness of the MLE of $\lambda$ instead of $\theta$. The $\log$-likelihood function $L=L_{O}(\tilde{\mathbf{w}} ; \lambda)$ based on a Type-II 
censored sample can be written as

$$
L=r \log \lambda+\sum_{i=1}^{r} \log \int \exp (-\lambda x) \mu_{\tilde{x}_{i}}(x) d x-(n-r) \lambda m_{(r)}
$$

Differentiating of (3.3) with respect to $\lambda$ yields

$$
\frac{\partial L}{\partial \lambda}=\frac{r}{\lambda}-\sum_{i=1}^{r} \frac{\int x \exp (-\lambda x) \mu_{\tilde{x}_{i}}(x) d x}{\int \exp (-\lambda x) \mu_{\tilde{x}_{i}}(x) d x}-(n-r) m_{(r)} .
$$

Let $g(\lambda)$ denote the function on the right-hand side of the expression in (3.4). Then it is easily seen that

$$
\lim _{\lambda \rightarrow 0} g(\lambda)=\infty
$$

Since the two terms $(n-r) m_{(r)}$ and $\int x \exp (-\lambda x) \mu_{\tilde{x}_{i}}(x) d x$ in (3.4) are both positive, we have

$$
\begin{aligned}
\lim _{\lambda \rightarrow \infty} g(\lambda) & =\lim _{\lambda \rightarrow \infty}\left[\frac{r}{\lambda}-\sum_{i=1}^{r} \frac{\int x \exp (-\lambda x) \mu_{\tilde{x}_{i}}(x) d x}{\int \exp (-\lambda x) \mu_{\tilde{x}_{i}}(x) d x}-(n-r) m_{(r)}\right] \\
& <\lim _{\lambda \rightarrow \infty} \frac{r}{\lambda}=0,
\end{aligned}
$$

$\forall \lambda \in(0, \infty)$. Therefore, the equation $g(\lambda)=0$ has at least one root in $(0, \infty)$. To prove that the root is unique, we consider the first derivative of $g, g^{\prime}(\lambda)$, given by

$$
g^{\prime}(\lambda)=-\frac{r}{\lambda^{2}}+\sum_{i=1}^{r} \frac{\partial^{2}}{\partial \lambda^{2}} \log \int \exp (-\lambda x) \mu_{\tilde{x}_{i}}(x) d x
$$

Now let

$$
\begin{gathered}
s(\lambda)=\exp (-\lambda x), \\
w_{i}(\lambda)=\int \exp (-\lambda x) \mu_{\tilde{x}_{i}}(x) d x
\end{gathered}
$$

Then $g^{\prime}(\lambda)$ can be written as

$$
g^{\prime}(\lambda)=-\frac{r}{\lambda^{2}}+\sum_{i=1}^{r} \frac{\partial^{2}}{\partial \theta^{2}} \log w_{i}(\theta)
$$

It is clearly that $s(\lambda)$ is a $\log$-concave function of $\lambda$, and this implies that $w_{i}(\lambda), i=1, \ldots, r$, are also log-concave in $\lambda$ (see Prekopa-Leindler inequality in the Appendix A). It follows that $g$ is a strictly decreasing function w.r.t. $\lambda$ and hence the equation $g(\lambda)=0$ has exactly one solution.

Since there is no closed form of the solution to the likelihood equation (3.2), an iterative numerical search can be used to obtain the MLE. In the following, we describe the Newton-Raphson method and the EM algorithm for determining the MLE of the parameter $\theta$. 


\subsubsection{Newton-Raphson algorithm}

Newton-Raphson algorithm is a direct approach for estimating the relevant parameters in a likelihood function. In this algorithm, the solution of the likelihood equation is obtained through an iterative procedure. Let $\theta^{(h)}$ be the parameter value from the $h$-th step. Then, at the $(h+1)-$ th step of iteration process, the updated parameter is obtained as

$$
\hat{\boldsymbol{\theta}}^{(h+1)}=\boldsymbol{\theta}^{(h)}-\frac{\left.\frac{\partial}{\partial \theta} L_{O}(\tilde{\mathbf{w}} ; \theta)\right|_{h}}{\left.\frac{\partial^{2}}{\partial \theta^{2}} L_{O}(\tilde{\mathbf{w}} ; \theta)\right|_{h}}
$$

where the notation $\left.A\right|_{h}$, for any partial derivative $A$, means the partial derivative evaluated at $\theta^{(h)}$. The second-order derivative of the log-likelihood with respect to the parameter, required for proceeding with the Newton-Raphson method, is obtained as follows.

$$
\begin{aligned}
& \frac{\partial^{2}}{\partial \theta^{2}} L_{O}(\tilde{\mathbf{w}} ; \theta)= \frac{r}{\theta^{2}}-2(n-r) \frac{m_{(r)}}{\theta^{3}} \\
&+\sum_{i=1}^{r}\left\{\frac{\left[\int\left(\frac{x^{2}}{\theta^{4}}-\frac{2 x}{\theta^{3}}\right) \exp \left(-\frac{x}{\theta}\right) \mu_{\tilde{x}_{i}}(x) d x\right]}{\int \exp \left(-\frac{x}{\theta}\right) \mu_{\tilde{x}_{i}}(x) d x}\right. \\
&\left.-\left[\frac{\int \frac{x}{\theta^{2}} \exp \left(-\frac{x}{\theta}\right) \mu_{\tilde{x}_{i}}(x) d x}{\int \exp \left(-\frac{x}{\theta}\right) \mu_{\tilde{x}_{i}}(x) d x}\right]^{2}\right\} .
\end{aligned}
$$

The iteration process then continues until convergence, i.e., until $\left|\theta^{(h+1)}-\theta^{(h)}\right|<\varepsilon$, for some prefixed $\varepsilon>0$. It should be pointed out that the second-order derivative of the $\log$-likelihood is required at every iteration in the Newton-Raphson method. Sometimes the calculation of the derivatives based on censored fuzzy data are complicated. Another viable alternative to the Newton-Raphson algorithm is the well-known EM algorithm. In the following, we discuss how that can be used to determine the MLE in this case.

Once the maximum likelihood estimate of $\theta$ is obtained, we can use the asymptotic normality of the MLEs to construct the approximate confidence interval. It is known that the asymptotic distribution of the MLE of $\theta$ is given by

$$
(\hat{\theta}-\theta) \rightarrow N(0, \operatorname{Var}(\theta)) .
$$

Here, the asymptotic variance $\operatorname{Var}(\theta)$ can be approximated by

$$
\operatorname{Var}(\theta) \approx \frac{1}{I(\hat{\theta})},
$$

where $I(\hat{\theta})$ denotes the observed Fisher information given by

$$
I(\hat{\boldsymbol{\theta}})=-\left.\frac{\partial^{2}}{\partial \theta^{2}} L_{O}(\tilde{\mathbf{w}} ; \theta)\right|_{\theta=\hat{\theta}}
$$

Remark 3.1. In the above proofs, we have exchanged integration and differentiation in (3.2), (3.4), and (3.7). Strict justification of these exchanges requires application of the dominated convergence theorem. To use this theorem, we note that the functions that we encounter in these relations can be readily verified to be bounded by Lebesgue integrable functions. Hence, for the cases that we 
considered, the dominated convergence theorem can be readily applied to exchange the order of integration and differentiation.

\subsubsection{EM algorithm}

Expectation Maximization (EM) algorithm has emerged as a very important tool for estimating the parameters involved in a model, especially when the available data are incomplete. Since the observed fuzzy data $\tilde{\mathbf{w}}$ can be seen as an incomplete specification of a complete data, the EM algorithm is applicable to obtain the maximum likelihood estimate of the parameter. First of all, denote the lifetime of the failed and censored units by $\mathbf{X}=\left(X_{1}, \ldots, X_{r}\right)$ and $\mathbf{Z}=\left(Z_{r+1}, \ldots, Z_{n}\right)$, respectively. The combination of $(\mathbf{X}, \mathbf{Z})=\mathbf{W}$ forms the complete lifetimes and the corresponding log-likelihood function is denoted by $L(\mathbf{W} ; \theta)$. In the following, we use the EM algorithm to determine the MLE of $\theta$.

The log-likelihood function based on the complete lifetime $\mathbf{W}$ becomes

$$
L(\mathbf{W} ; \theta)=-n \log \theta-\frac{1}{\theta}\left[\sum_{i=1}^{r} x_{i}+\sum_{j=r+1}^{n} z_{j}\right] .
$$

To perform the E-step of the EM algorithm, we need to compute the conditional expectation of the complete-data log likelihood conditionally on the observed data $\tilde{\mathbf{w}}$, using the current fit $\theta^{(h)}$ of the parameter $\theta$ :

$$
E_{\boldsymbol{\theta}^{(h)}}(L(\mathbf{W} ; \theta) \mid \tilde{\mathbf{w}})=-n \log \theta-\frac{1}{\theta}\left[\sum_{i=1}^{r} E_{\theta^{(h)}}\left(X_{i} \mid \tilde{x}_{i}\right)+\sum_{j=r+1}^{n} E_{\theta^{(h)}}\left(Z_{j} \mid \tilde{z}_{j}\right)\right],
$$

where

$$
\begin{gathered}
E_{\theta^{(h)}}\left(X_{i} \mid \tilde{x}_{i}\right)=\frac{\int x \exp \left(-\frac{x}{\left(\theta^{(h)}\right)}\right) \mu_{\tilde{x}_{i}}(x) d x}{\int \exp \left(-\frac{x}{\left(\theta^{(h)}\right)}\right) \mu_{\tilde{x}_{i}}(x) d x}, \quad i=1, \ldots, r, \\
E_{\theta^{(h)}}\left(Z_{j} \mid \tilde{z}_{j}\right)=m_{(r)}+\theta^{(h)}, \quad j=r+1, \ldots, n .
\end{gathered}
$$

The M-step then consists in finding $\theta^{(h+1)}$ which maximizes $E_{\theta^{(h)}}(\log L(\mathbf{W} ; \theta) \mid \tilde{\mathbf{w}})$. This is easily achieved by solving the likelihood equation. From

$$
\frac{\partial}{\partial \theta} E_{\theta^{(h)}}(\log L(\mathbf{W} ; \theta) \mid \tilde{\mathbf{w}})=0
$$

we get

$$
\hat{\boldsymbol{\theta}}^{(h+1)}=\frac{1}{n}\left[\sum_{i=1}^{r} E_{\boldsymbol{\theta}^{(h)}}\left(X_{i} \mid \tilde{x}_{i}\right)+(n-r)\left(m_{(r)}+\boldsymbol{\theta}^{(h)}\right)\right] .
$$

The MLE of $\theta$ can be obtained by repeating the E- and M-steps until the difference $L_{O}\left(\tilde{\mathbf{w}} ; \theta^{(h+1)}\right)-$ $L_{O}\left(\tilde{\mathbf{w}} ; \theta^{(h)}\right)$ becomes smaller than some arbitrary small amount. Due to the result of the Theorem 
3.1, it is then evident that the EM algorithm and the Newton_Raphson method would converge to the same value. The maximum likelihood estimate of $\theta$ via EM algorithm is thereafter refereed as " $\hat{\theta}_{E M}$ "in this paper.

\subsection{Method of moments}

Let $X$ be random variable which has the Exponential distribution with pdf given by (2.1). It is well-known that the $k$ th moment of the Exponential model with parameter $\theta$ is

$$
E\left(X^{k}\right)=\theta^{k} \Gamma(k+1) .
$$

Equating the first sample moment to the corresponding population moment, the following equation can be used to find the estimate of moment method.

$$
\theta=\frac{1}{n}\left[\sum_{i=1}^{r} \frac{\int x \exp \left(-\frac{x}{\theta}\right) \mu_{\tilde{x}_{i}}(x) d x}{\int \exp \left(-\frac{x}{\theta}\right) \mu_{\tilde{x}_{i}}(x) d x}+(n-r)\left(m_{(r)}+\theta\right)\right] .
$$

Since the closed form of the solution to Eq. (3.12) could not be obtained, an iterative numerical process to obtain the parameter estimate is described as follows:

1. Let the initial estimate of $\theta$, say $\theta^{(0)}$ with $h=0$.

2. In the $(h+1)$ th iteration, we compute new estimate of $\theta$, say $\theta^{(h+1)}$ from:

$$
\boldsymbol{\theta}^{(h+1)}=\frac{1}{n}\left[\sum_{i=1}^{r} \frac{\int x \exp \left(-\frac{x}{\theta^{(h)}}\right) \mu_{\tilde{x}_{i}}(x) d x}{\int \exp \left(-\frac{x}{\theta^{(h)}}\right) \mu_{\tilde{x}_{i}}(x) d x}+(n-r)\left(m_{(r)}+\theta^{(h)}\right)\right] .
$$

3. Checking convergence, if the convergence occurs then the current $\theta^{(h+1)}$ is the estimate of $\theta$ by the method of moments; Otherwise set $h=h+1$ and go to Step 2. The resultant estimate of $\theta$ is thereafter refereed as " $\hat{\theta}_{M M}$ "in this paper.

\subsection{Bayesian estimation}

In this subsection we consider the Bayes estimation of the unknown parameter. We first reparameterize the model as follows $\lambda=\frac{1}{\theta}$. As conjugate prior for $\lambda$, we take the $\operatorname{Gamma}(a, b)$ density with pdf given by

$$
\pi(\lambda)=\frac{b^{a}}{\Gamma(a)} \lambda^{a-1} \exp (-\lambda b), \quad \lambda>0,
$$

where $a>0$ and $b>0$. Based on the new parametrization, the posterior density function of $\lambda$ given the data can be written as follows.

$$
\pi(\lambda \mid \tilde{\mathbf{w}}) \propto \frac{\pi(\lambda) \ell(\tilde{\mathbf{w}} ; \lambda)}{\int_{0}^{\infty} \pi(\lambda) \ell(\tilde{\mathbf{w}} ; \lambda) d \lambda},
$$


where

$$
\ell(\tilde{\mathbf{w}} ; \lambda)=\lambda^{r} \exp \left[-(n-r) \lambda m_{(r)}\right] \prod_{i=1}^{r} \int \exp (-\lambda x) \mu_{\tilde{x}_{i}}(x) d x
$$

is the likelihood function based on the Type-II censored sample. Then, under a squared error loss, the Bayes estimate of any function of $\lambda$, say $g(\lambda)$, is

$$
E(g(\lambda) \mid \tilde{\mathbf{w}})=\frac{\int_{0}^{\infty} g(\lambda) \pi(\lambda) \ell(\tilde{\mathbf{w}} ; \lambda) d \lambda}{\int_{0}^{\infty} \pi(\lambda) \ell(\tilde{\mathbf{w}} ; \lambda) d \lambda}=\frac{\int_{0}^{\infty} g(\lambda) e^{Q(\lambda)} d \lambda}{\int_{0}^{\infty} e^{Q(\lambda)} d \lambda},
$$

where $Q(\lambda)=\ln \pi(\lambda)+\ln \ell(\tilde{\mathbf{w}} ; \lambda) \equiv \rho(\lambda)+L(\lambda)$. Since the Eq. (18) can not be obtained analytically, we adopt Lindley's approximation to compute the Bayes estimate of $\theta$. Lindley [12] first proposed his procedure to approximate the ratio of two integrals such as (3.16). For the one-parameter case, Lindley's approximation of (3.16) is the form

$$
g(\lambda)+\frac{1}{2} g_{11} \tau_{11}+\rho_{1} g_{1} \tau_{11}+\frac{1}{2} L_{3} \tau_{11}^{2} g_{1}
$$

where

$$
\begin{gathered}
g_{1}=\frac{d g(\lambda)}{d \lambda}, g_{11}=\frac{d^{2} g(\lambda)}{d \lambda^{2}}, \rho_{1}=\frac{d \rho(\lambda)}{d \lambda} \\
L_{3}=\frac{\partial^{3} L(\lambda)}{\partial \lambda^{3}}, \tau_{11}=\left[-\frac{\partial^{2} L(\lambda)}{\partial \lambda^{2}}\right]^{-1} .
\end{gathered}
$$

Evaluating all the expressions in (3.17) at the MLE of $\lambda$ produces the approximation $\hat{g}_{B}$ to (3.16). In our case, we have

$$
L(\lambda)=r \log \lambda+\sum_{i=1}^{r} \log \int \exp (-\lambda x) \mu_{\tilde{x}_{i}}(x) d x-(n-r) \lambda m_{(r)} .
$$

The MLE of $\lambda$ is the solution of the equation $\frac{\partial L(\lambda)}{\partial \lambda}=0$.

Now, to apply Lindley's form in (3.17), we first obtain

$$
\tau_{11}=-\frac{r}{\hat{\lambda}^{2}}+\sum_{i=1}^{r}\left\{\frac{\int x^{2} \exp (-\hat{\lambda} x) \mu_{\tilde{x}_{i}}(x) d x}{\int \exp (-\hat{\lambda} x) \mu_{\tilde{x}_{i}}(x) d x}-\left[\frac{\int x \exp (-\hat{\lambda} x) \mu_{\tilde{x}_{i}}(x) d x}{\int \exp (-\hat{\lambda} x) \mu_{\tilde{x}_{i}}(x) d x}\right]^{2}\right\}
$$


and

$$
\begin{aligned}
L_{3}=\frac{2 r}{\hat{\lambda}^{3}}-\sum_{i=1}^{r}\left\{\frac{\int x^{3} \exp (-\hat{\lambda} x) \mu_{\tilde{x}_{i}}(x) d x}{\int \exp (-\hat{\lambda} x) \mu_{\tilde{x}_{i}}(x) d x}\right. & {\left[\int x^{2} \exp (-\hat{\lambda} x) \mu_{\tilde{x}_{i}}(x) d x\right]\left[x \exp (-\hat{\lambda} x) \mu_{\tilde{x}_{i}}(x) d x\right] } \\
-\left[\int \exp (-\hat{\lambda} x) \mu_{\tilde{x}_{i}}(x) d x\right]^{2} & \frac{\left[\mu^{2}\right.}{\int \sum_{i=1}}\left\{\frac{\int x \exp (-\hat{\lambda} x) \mu_{\tilde{x}_{i}}(x) d x}{\int \exp (-\hat{\lambda} x) \mu_{\tilde{x}_{i}}(x) d x}\right. \\
& \left.\times\left(\frac{\int x^{2} \exp (-\hat{\lambda} x) \mu_{\tilde{x}_{i}}(x) d x}{\int \exp (-\hat{\lambda} x) \mu_{\tilde{x}_{i}}(x) d x}-\left[\frac{\int x \exp (-\hat{\lambda} x) \mu_{\tilde{x}_{i}}(x) d x}{\int \exp (-\hat{\lambda} x) \mu_{\tilde{x}_{i}}(x) d x}\right]^{2}\right)\right\}
\end{aligned}
$$

The approximate Bayes of $\theta$, say $\hat{\theta}_{B}$, for the squared error loss function is the posterior mean of $g(\lambda)=\frac{1}{\lambda}$, which is by (3.17) as follows.

$$
\hat{\theta}_{B}=\frac{1}{\hat{\lambda}}+\frac{\tau_{11}}{\hat{\lambda}^{3}}-\frac{2 \rho_{1} \tau_{11}+L_{3} \tau_{11}^{2}}{2 \hat{\lambda}^{2}}
$$

where $\rho_{1}=\frac{a-1}{\hat{\lambda}}-b$.

\subsection{Computational approach estimation method}

In this subsection we propose a new parameter estimation procedure called 'CAE'. Although the maximum likelihood estimate obtained in the preceding section is preferable, its computation requires repeated evaluation of E- and M-steps until convergence occurs. On the other hand, the CAE method provides not only the computational ease but also reasonable mean squared error. This finding is further discussed in Section 4.

Suppose $\tilde{x}_{i}=\left(\alpha_{i}, m_{i}, \beta_{i}\right), \quad i=1, \ldots, r$, be the observed fuzzy lifetimes under Type-II censoring from exponential distribution with unknown parameter $\theta$. Grzegorzewski and Hryniewicz [7] considered the generalization of exponential model which admits vagueness in lifetimes. They obtained a fuzzy estimator of the mean lifetime $\theta$ in the presence of vague lifetimes. However, in most applications, crisp results are required instead of fuzzy ones. So, we propose the following computational approach to obtain a crisp value as an estimate of $\theta$.

Step 1: Order the means of fuzzy numbers as $m_{(1)}<m_{(2)}<\ldots<m_{(r)}$.

Step 2: Obtain the fuzzy mean value, say $\overline{\tilde{x}}$, of the fuzzy numbers by using (2.2).

Step 3: Convert the fuzzy number $\overline{\tilde{x}}$ into a real value by using the center of gravity defuzzification technique (see in the Appendix B). Denote this defuzzified value by $\widetilde{\widetilde{x}}^{*}$.

Step 4: The new estimate of $\theta$ is then computed as:

$$
\widetilde{\widetilde{\theta}}=\widetilde{\widetilde{x}}^{*}+\left(\frac{n}{r}-1\right) m_{(r)} \text {. }
$$

The resultant estimate of $\theta$ via this approach is thereafter refereed as " $\hat{\theta}_{C A}$ "in this paper. 


\section{Numerical Study}

In this section, we present a Monte Carlo simulation study and one example to illustrate the methods of inference developed in this paper. First, for fixed $\theta=1$ and different choices of $n$ and $r$, we have generated Type-II censored samples from Exponential distribution, using the method proposed by Aggarwala and Balakrishnan [1], as follows.

(1) Generate $Z_{i}$ from $U(0,1)$ for $i=1, \ldots, r$.

(2) Set $V_{i}=Z_{i}^{\frac{1}{a_{i}}}, a_{i}=i+n-r, i=1, \ldots, r$.

(3) Set $U_{i}=1-V_{r-i+1} V_{r-i+2} \ldots V_{r}, i=1, \ldots, r$.

(4) Thus, $X_{i}=F^{-1}\left(U_{i}\right), i=1, \ldots, r$, is the desired Type-II censored sample from the Exponential distribution.

Then each realization of $\mathbf{x}$ was fuzzified using the method proposed by Pak et al. [15], and the estimate of $\theta$ for the fuzzy sample were computed using the four methods presented in the preceding section. We have used the initial estimate of $\theta$ for iterative progresses of Newton-Raphson method, EM algorithm and moment method to be $\theta^{(0)}=\frac{1}{r} \sum_{i=1}^{r} x_{i}$. For computing the Bayes estimator, we have assumed that $\lambda$ has $\operatorname{Gamma}(a, b)$ prior. To make the comparison meaningful, it is assumed that the prior is non-informative, and they are $a=b=0$. The average values and mean squared errors of the estimates, the average number of iterations needed for achieving convergence, based on 1000 replication are presented in Table 1.

In viewing the table, we find that using Newton-raphson or EM algorithm for the computation of MLE give similar estimation results, but Newton-Raphson method needs smaller iterations for convergence. The performance of MLE and MME in terms of AVs and MSEs are quite similar to each other. Among the four procedures developed here, the CAE method produces estimates with smallest MSE and then followed by MLE and MME and then the Bayes procedure. In all the cases, it is observed that for fixed $n$ as $m$ increases, the MSEs of the estimates decrease as expected. Since these four procedures for estimating the mean parameter $\theta$ of Exponential distribution have different features, we let users to decide based on their preferences.

Example 1. To demonstrate the application of proposed methods, let us consider the following life-testing experiment in which $n=23$ identical batteries are placed on test. The unknown lifetime $x_{i}$ of battery $i$ may be regarded as a realization of a random variable $X_{i}$, induced by random sampling from a total population of batteries, which is distributed as Exponential by an unknown mean parameter of $\theta$. A tested battery may be considered as failed, or -strictly speaking- as nonconforming, when at least one value of its parameters falls beyond specification limits. In practice, however, we don't have the possibility to measure all parameters and are not able to define precisely the moment of a failure. So, the observed lifetimes (in 100h) are reported in the form of lower and upper bounds, as well as a point estimate which are as follows.

DATA SET:

$$
\begin{aligned}
& (2.90,3.63,3.99),(5.24,6.55,7.20),(6.56,8.20,9.02),(7.14,8.93,9.82), \\
& (11.60,14.51,15.96),(12.14,15.18,16.69),(12.65,15.82,17.40),(13.24,16.56,18.21), \\
& (13.67,17.09,18.79),(13.88,17.36,19.09),(15.64,19.56,21.51),(17.05,21.32,23.45), \\
& (17.40,21.76,23.93),(17.80,22.26,24.48),(19.01,23.77,26.14),(19.34,24.18,26.59), \\
& (23.13,28.92,31.81),(23.34,29.18,32.09),(26.07,32.59,35.84),(30.29,37.87,41.65), \\
& (43.97,54.97,60.46),(48.09,60.12,66.13),(73.48,91.86,98.04) .
\end{aligned}
$$


N.B. Khoolenjani and F. Shahsanaie

Table 1. Averages values and mean squared errors of the estimates, average number of iterations (AI) needed for convergence.

\begin{tabular}{|c|c|c|c|c|c|c|c|c|c|}
\hline$n$ & $r$ & $\hat{\theta}_{N R}$ & $\hat{\theta}_{E M}$ & $\hat{\theta}_{M M}$ & $\hat{\theta}_{B}$ & $\hat{\theta}_{C A}$ & $A I_{N R}$ & $A I_{E M}$ & $A I_{M M}$ \\
\hline \multirow[t]{6}{*}{15} & \multirow[t]{2}{*}{5} & 0.961 & 0.960 & 0.961 & 0.973 & 0.982 & \multirow[t]{2}{*}{9.183} & \multirow[t]{2}{*}{15.621} & \multirow[t]{2}{*}{33.091} \\
\hline & & $(0.305)$ & (0.304) & $(0.305)$ & $(0.331)$ & $(0.300)$ & & & \\
\hline & \multirow[t]{2}{*}{8} & 1.033 & 1.032 & 1.033 & 1.037 & 1.039 & \multirow[t]{2}{*}{7.886} & \multirow[t]{2}{*}{9.064} & \multirow[t]{2}{*}{18.879} \\
\hline & & $(0.211)$ & $(0.210)$ & $(0.211)$ & $(0.221)$ & $(0.202)$ & & & \\
\hline & \multirow[t]{2}{*}{12} & 1.013 & 1.012 & 1.013 & 1.027 & 1.007 & \multirow[t]{2}{*}{5.139} & \multirow[t]{2}{*}{7.543} & \multirow[t]{2}{*}{11.293} \\
\hline & & $(0.142)$ & $(0.141)$ & $(0.142)$ & $(0.156)$ & (0.114) & & & \\
\hline \multirow[t]{6}{*}{20} & \multirow[t]{2}{*}{5} & 1.030 & 1.029 & 1.030 & 1.039 & 1.049 & \multirow[t]{2}{*}{10.052} & \multirow[t]{2}{*}{21.226} & \multirow[t]{2}{*}{45.076} \\
\hline & & $(0.238)$ & $(0.237)$ & $(0.238)$ & $(0.246)$ & $(0.235)$ & & & \\
\hline & \multirow[t]{2}{*}{10} & 1.022 & 1.021 & 1.022 & 1.034 & 1.019 & \multirow[t]{2}{*}{8.155} & \multirow[t]{2}{*}{9.932} & \multirow[t]{2}{*}{20.352} \\
\hline & & (0.110) & (0.110) & (0.110) & (0.119) & (0.104) & & & \\
\hline & \multirow[t]{2}{*}{15} & 0.995 & 0.995 & 0.995 & 1.015 & 1.009 & \multirow[t]{2}{*}{6.647} & \multirow[t]{2}{*}{5.985} & \multirow[t]{2}{*}{12.259} \\
\hline & & $(0.100)$ & $(0.100)$ & $(0.100)$ & (0.117) & $(0.086)$ & & & \\
\hline \multirow[t]{6}{*}{30} & 10 & 0.974 & 0.973 & 0.974 & 0.989 & 0.982 & \multirow[t]{2}{*}{9.159} & \multirow[t]{2}{*}{15.446} & \multirow[t]{2}{*}{32.995} \\
\hline & & $(0.118)$ & $(0.117)$ & $(0.118)$ & $(0.126)$ & $(0.115)$ & & & \\
\hline & \multirow[t]{2}{*}{15} & 0.998 & 0.997 & 0.998 & 1.018 & 0.999 & \multirow[t]{2}{*}{8.193} & 9.938 & 20.259 \\
\hline & & $(0.066)$ & $(0.065)$ & $(0.066)$ & $(0.072)$ & $(0.057)$ & & & \\
\hline & 20 & 1.006 & 1.005 & 1.006 & 1.027 & 1.017 & 7.117 & 6.898 & 13.872 \\
\hline & & $(0.063)$ & $(0.063)$ & $(0.063)$ & $(0.070)$ & $(0.051)$ & & & \\
\hline 50 & 15 & 1.008 & 1.007 & 1.008 & 1.025 & 1.023 & 9.917 & 17.45 & 37.002 \\
\hline & & $(0.089)$ & $(0.088)$ & $(0.089)$ & $(0.095)$ & $(0.086)$ & & & \\
\hline & 20 & 1.004 & 1.003 & 1.004 & 1.019 & 1.028 & 9.029 & 12.842 & 26.975 \\
\hline & & $(0.058)$ & $(0.058)$ & $(0.058)$ & $(0.062)$ & $(0.052)$ & & & \\
\hline & 30 & 0.990 & 0.990 & 0.990 & 0.999 & 0.995 & 7.65 & 7.97 & 16.013 \\
\hline & & $(0.036)$ & $(0.036)$ & $(0.036)$ & $(0.039)$ & $(0.034)$ & & & \\
\hline
\end{tabular}

In our approach, each triple is modeled by a triangular fuzzy number $\tilde{x}_{i}$, and is interpreted as a possibility distribution related to an unknown value $x_{i}$, itself a realization of a random variable $X_{i}$. Randomness arises from the selection of objects from the total population of batteries. In contrast, fuzziness arises from the limited ability of the observer to describe the moment of a failure using numbers, which is not influenced by random factors. Suppose that the last 7 lifetimes out of $n$ inspection items are missing, then we can only obtain a Type-II censored sample

$(2.90,3.63,3.99),(5.24,6.55,7.20),(6.56,8.20,9.02),(7.14,8.93,9.82)$,

$(11.60,14.51,15.96),(12.14,15.18,16.69),(12.65,15.82,17.40),(13.24,16.56,18.21)$,

$(13.67,17.09,18.79),(13.88,17.36,19.09),(15.64,19.56,21.51),(17.05,21.32,23.45)$,

$(17.40,21.76,23.93),(17.80,22.26,24.48),(19.01,23.77,26.14),(19.34,24.18,26.59)$. 
From these data, and using the starting value $\theta^{(0)}=16.04$, which is the estimate of the parameter computed over the centers of each fuzzy numbers, the final MLE of $\theta$ is found from NewtonRaphson and EM algorithm to be $\hat{\theta}_{N R}=26.29$ and $\hat{\theta}_{E M}=26.28$, respectively. Based on Lindley's approximation, the Bayes estimate of $\theta$ under squared error loss function becomes $\hat{\theta}_{B}=26.93$. Also the estimates from the CAE and moment method are $\hat{\theta}_{C A}=26.35$ and $\hat{\theta}_{M M}=26.29$, respectively.

\section{Conclusions}

Although the problem of estimation of exponential mean parameter based on the censored data has been studied extensively, traditionally it is assumed that the data available are performed in exact numbers. In real world situations, however, we deal with non-precise (fuzzy) data. Therefore, the conventional procedures used for estimating the unknown parameter will have to be adopted to the new situation. In this paper we have proposed different procedures for estimating the exponential distribution parameter under Type-II censoring when the lifetime observations are fuzzy numbers. They are maximum likelihood estimation, estimation of method moments, Bayesian estimation and computational approach estimation method. We have then carried out a simulation study to assess the performance of all these procedures. Based on the results of the simulation study, we see clearly that, maximum likelihood estimator (via any of the two processes mentioned in the paper) and "MME"give the same parameter estimates as shown by AV and MSE in Table 1. The "CAE"has slightly better MSE compared with all the estimators considered here. In all the cases, it is observed that as the effective sample size increases the performances in terms of MSE become better. The study of the applicability of the proposed approach in estimating the parameters of exponential distribution under the other censoring schemes such as Hybrid type-II and Hybrid progressive typeII censoring are possible topics for further research.

\section{Appendix A. Prekopa-Leindler inequality}

In mathematics, the Prekopa-Leindler inequality is an integral inequality closely related to the reverse Young's inequality, the Brunn-Minkowski inequality and a number of other important and classical inequalities in analysis.

Statement of the inequality (see [6]):

Let $0<\lambda<1$ and let $f, g$ and $h$ be non-negative integrable functions defined on $\mathbb{R}^{n}$ satisfying

$$
h((1-\lambda) x+\lambda y) \geq f(x)^{1-\lambda} g(y)^{\lambda}
$$

for all $x, y \in \mathbb{R}^{n}$. Then

$$
\int_{\mathbb{R}^{n}} h(x) d x \geq\left(\int_{\mathbb{R}^{n}} f(x) d x\right)^{1-\lambda}\left(\int_{\mathbb{R}^{n}} g(x) d x\right)^{\lambda} .
$$

\section{Appendix B. Center of gravity defuzzification method}

Defuzzyfication is the opposite process to the essence of idea of fuzzy sets. Moreover, defuzzyfication is the last step on fuzzy control system and fuzzy reasoning system. Finally, defuzzyfication operation reduces, fuzzy number to a single, crisp, numerical value, result carries the best information and makes kind of synthesis about this fuzzy number. A common and useful defuzzification 
technique is the center of gravity method. The center of gravity defuzzification technique can be expressed as ( [18], pp. 99)

$$
z^{*}=\frac{\int z \mu_{\tilde{C}}(z) d z}{\int \mu_{\tilde{C}}(z) d z}
$$

where $z^{*}$ is the defuzzified output, $\mu_{\tilde{C}}(z)$ is the membership function of the fuzzy set $\tilde{C}$.

\section{Acknowledgement}

The authors are also grateful to anonymous reviewers whose valuable comments and suggestions led to improvement of the manuscript.

\section{References}

[1] R., Aggarwala, and N., Balakrishnan, Some properties of progressively censored order statistics from arbitrary and uniform distributions with applications to inference and simulation. Journal of Statistical planning and inference, 70, (1998), 35-49.

[2] N., Balakrishnan, and D., Han, Exact inference for a simple step-stress model with competing risks for failure from exponential distribution under Type-II censoring. Journal of Statistical Planning and Inference, 138(12), (2008), 172-4186.

[3] P.S., Chan, H.K.T., Ng, N., Balakrishnan, and Q., Zhou, Point and interval estimation for extreme-value regression model under Type-II censoring. Computational Statistics \& Data Analysis, 52(8), (2008), 4040-4058.

[4] R., Coppi, M.A., Gil, and H.A.L., Kiers, The fuzzy approach to statistical analysis. Computational Statistics and Data Analysis, 51(1), (2006), 114.

[5] D., Dubois, and H., Prade, Fuzzy Sets and Systems: Theory and Applications, Academic Press, New York, (1980).

[6] R.J., Gardner, The Brunn-Minkowski inequality ,Bulletin of the American mathematical society. 39(3), (2002), 355-405.

[7] P., Grzegorzewski, and O., Hryniewicz, Computing with words and life data, International Journal of Applied Mathematics and Computer Science, 12(3), (2002), 337-345.

[8] H., Huang, M., Zuo, and Z., Sun, Bayesian reliability analysis for fuzzy lifetime data. Fuzzy Sets and Systems, 157, (2006), 16741686.

[9] G., Iliopoulos, and N., Balakrishnan, Exact likelihood inference for Laplace distribution based on TypeII censored samples, Journal of Statistical Planning and Inference, 141(3), (2011), 1224-1239.

[10] D., Kundu, and M. Z., Raqab, Bayesian inference and prediction of order statistics for a Type-II censored Weibull distribution. Journal of Statistical Planning and Inference, 142(1), (2012), 41-47.

[11] A.J., Lemonte, and S.L.P., Ferrari, Testing hypotheses in the BirnbaumSaunders distribution under typeII censored samples. Computational Statistics \& Data Analysis, 55(7), (2011), 2388-2399.

[12] D.V., Lindley, Approximate Bayesian method. Trabajos de Estadistica, 31, (1980), 223-237.

[13] H.K.T., Ng, D., Kundu, and N., Balakrishnan, Point and interval estimation for the two-parameter Birnbaum-Saunders distribution based on Type-II censored samples, Computational Statistics \& Data Analysis, 50(11), (2006), 3222-3242

[14] A., Pak, G.H., Parham, and M., Saraj, On estimation of Rayleigh scale parameter under doubly Type-II censoring from imprecise data. Journal of Data Science, 11, (2013), 303-320.

[15] A., Pak, G.H., Parham, and M., Saraj, Inferences on the Competing Risk Reliability Problem for Exponential Distribution Based on Fuzzy Data. IEEE Transactions on reliability, 63(1), (2014), 2-13.

[16] H., Panahi, and S., Asadi, Estimation of the Weibull Distribution Based on Type-II Censored Samples, Applied Mathematical Sciences, 52(5), (2011), 2549-2558.

[17] L., Qian, The Fisher information matrix for a three-parameter exponentiated Weibull distribution under type II censoring. Statistical Methodology, 9(3), (2012), 320329. 
[18] T. J., Ross, Fuzzy Logic with Engineering Applications, John Wiley \& Sons, Ltd, (2009).

[19] X., Sun, X., Zhou, and J., Wang, Confidence intervals for the scale parameter of exponential distribution based on Type II doubly censored samples. Journal of Statistical Planning and Inference, 138(7), (2008), 2045-2058.

[20] L. A., Zadeh, Probability measures of fuzzy events, Journal of Mathematical Analysis and Applications, 10, (1968), 421-427.

[21] H. J., Zimmermann, Fuzzy set teory and its application, Kluwer, Dordrecht, (1991). 